DOI: $10.15593 / 2499-9873 / 2020.4 .01$

УДК 519.21

\author{
А.В. Ганичева \\ Тверская государственная сельскохозяйственная \\ академия, Тверь, Россия \\ ОЦЕНКА ЧИСЛА СЛАГАЕМЫХ \\ ЦЕНТРАЛЬНОЙ ПРЕДЕЛЬНОЙ ТЕОРЕМЫ
}

\begin{abstract}
Исследуется задача оценки числа слагаемых случайных величин для суммарного нормального закона распределения или выборочной средней, имеющей нормальное распределение. Центральная предельная теорема позволяет решать многие сложные прикладные задачи, используя развитый математический аппарат нормального распределения вероятностей. В противном случае пришлось бы оперировать свертками распределений, которые в явном виде вычисляются в редких случаях.

Целью данной работы является теоретическая оценка числа слагаемых центральной предельной теоремы, необходимых для того, чтобы сумма или выборочная средняя имели нормальный закон распределения вероятностей. Доказаны две теоремы и два следствия из них. Для доказательства теорем использован метод характеристических функций. Первая теорема формулирует условия, при которых средняя выборочная независимых слагаемых будет иметь с заданной точностью нормальный закон распределения. Следствие из первой теоремы определяет нормальное распределение для суммы независимых случайных величин в условиях теоремы 1. Вторая теорема определяет условия нормального распределения для средней выборочной независимых случайных величин, математические ожидания которых попадают в один и тот же интервал, дисперсии также попадают в один и тот же интервал. Следствие из второй теоремы определяет нормальное распределение для суммы независимых случайных величин в условиях теоремы 2. По формульным соотношениям, доказанным в теореме 1, рассчитана таблица необходимого числа слагаемых в центральной предельной теореме для обеспечения заданной точности приближения распределения значений средней выборочной к нормальному закону распределения. Построен график данной зависимости. Зависимость хорошо аппроксимируется полиномом шестой степени.

Полученные в статье несложные с точки зрения проведения вычислений соотношения и доказанные теоремы позволяют управлять процессом тестирования для оценки знаний студентов. Они дают возможность определять число экспертов при принятии коллективных решений в экономике и системах организационного управления, проводить оптимальный выборочный контроль качества выпускаемой продукции, осуществлять проведение нужного количества наблюдений и обоснованную диагностику в медицине.

Ключевые слова: случайная величина, нормальный закон распределения, выборочная средняя, сумма ряда, дисперсия, характеристическая функция, ряд Маклорена, точность, относительная погрешность, интервал.
\end{abstract}


A.V. Ganicheva

Tverskaya State Agricultural Academy, Tver, Russian Federation

\section{ESTIMATION OF THE NUMBER OF SUMMANDS OF THE CENTRAL LIMIT THEOREM}

The problem of estimating the number of summands of random variables for a total normal distribution law or a sample average with a normal distribution is investigated. The Central limit theorem allows us to solve many complex applied problems using the developed mathematical apparatus of the normal probability distribution. Otherwise, we would have to operate with convolutions of distributions that are explicitly calculated in rare cases.

The purpose of this paper is to theoretically estimate the number of terms of the Central limit theorem necessary for the sum or sample average to have a normal probability distribution law. The article proves two theorems and two consequences of them. The method of characteristic functions is used to prove theorems. The first theorem States the conditions under which the average sample of independent terms will have a normal distribution law with a given accuracy. The corollary of the first theorem determines the normal distribution for the sum of independent random variables under the conditions of theorem 1. The second theorem defines the normal distribution conditions for the average sample of independent random variables whose mathematical expectations fall in the same interval, and whose variances also fall in the same interval. The corollary of the second theorem determines the normal distribution for the sum of independent random variables under the conditions of theorem 2 . According to the formula relations proved in theorem 1, a table of the required number of terms in the Central limit theorem is calculated to ensure the specified accuracy of approximation of the distribution of the values of the sample average to the normal distribution law. A graph of this dependence is constructed. The dependence is well approximated by a polynomial of the sixth degree.

The relations and proved theorems obtained in the article are simple, from the point of view of calculations, and allow controlling the testing process for evaluating students ' knowledge. They make it possible to determine the number of experts when making collective decisions in the economy and organizational management systems, to conduct optimal selective quality control of products, to carry out the necessary number of observations and reasonable diagnostics in medicine.

Keywords: random variable, normal distribution law, sample average, series sum, variance, characteristic function, Maclaurin's series, accuracy, relative error, interval.

\section{Введение}

Предельные теоремы составляют основу современной теории вероятностей. Среди них особое место по важности практического применения занимает центральная предельная теорема (ЦПТ). Она позволяет решать многие задачи в экономических, социальных, технических, военных, медицинских, биологических системах, где рассматриваются суммы случайных величин.

Примерами применения ЦПТ являются:

1) обоснование результатов измерений и наблюдений;

2) моделирование действия многих факторов на изучаемый процесс или явление;

3) статистическая теория принятия решений;

4) оценка рисков, управление рисками; 
5) организация страхования;

6) выборочный контроль и оценка качества продукции;

7) социологические исследования.

В научных публикациях рассматриваются преимущественно две проблемы ЦПТ: асимптотическое поведение распределений суммы случайных величин, например в работах [1-3], и скорость сходимости к предельному закону [4-6]. Новыми направлениями в данной области знаний являются исследование сумм зависимых случайных величин и функций от них [7], обоснование многомерной центральной предельной теоремы для зависимых случайных векторов [8], рассмотрение предельных теорем для случайных матриц с зависимыми элементами [9]. Методология проверки адекватности центральной предельной теоремы на основе связи этой теоремы и теоремы Бернулли изложена в статье [10].

Один важный для практики вопрос не нашел должного простого теоретического обоснования. Речь идет об оценке числа слагаемых центральной предельной теоремы так, чтобы сумма была распределена по нормальному закону. Эта задача решается в работах [11-13] путем моделирования на больших наборах данных. В работе [11] отмечается, что при использовании трех слагаемых график плотности вероятности начинает приближаться к нормальному; при 30 и более слагаемых график плотности вероятности близок к теоретическому. Дальнейшее увеличение не приводит к существенным изменениям характеристик.

Целью данной работы является теоретическая оценка числа слагаемых центральной предельной теоремы, необходимых для того, чтобы сумма имела нормальный закон распределения вероятностей.

\section{1. Постановка задачи оценки числа слагаемых центральной предельной теоремы}

В прикладной области часто возникает задача оценки числа слагаемых случайных величин для суммарного нормального закона распределения или выборочной средней, имеющей нормальное распределение. Это может быть оценка необходимого числа вопросов для проверки знаний обучаемых при тестировании, определение числа экспертов для формирования обоснованной коллективной оценки, задание числа выборочных проверок при контроле качества продукции и т.д. 
В теоретических аспектах это формулируется следующим образом: для достаточно большого числа слагаемых $n$ при определенных условиях сумма или средняя выборочная будет иметь нормальное распределение. Однако это звучит очень неопределенно. В работах $[14,15]$ конкретизируется, что, как показывает практика, уже при $n=10$ суммарное распределение будет нормальным. В связи с этим возникает задача оценки числа $n$, например в виде функциональной зависимости $n$ от параметров, связанных с описанием закона распределения суммы или средней выборочной.

\section{2. Основные теоремы и следствия}

Докажем следующее утверждение.

Teорема 1. При сложении $n$ независимых слагаемых случайных величин, имеющих одинаковые математические ожидания $m$ и дисперсии $\sigma^{2}$, их средняя выборочная будет иметь с точностью $\varepsilon$ нормальный закон распределения при

$$
n \geq n_{0}=\max \left\{\lfloor\sqrt{4,5}\rfloor+1,\left\lfloor\frac{2,16}{\sqrt[3]{\varepsilon}}\right\rfloor+1\right\} .
$$

Здесь символ $\lfloor a\rfloor$ означает целую часть числа $a$.

Доказательство. Оценим значение $n_{0}$ с использованием метода характеристических функций. Не нарушая общности, рассмотрим непрерывные случайные величины.

Обозначим через $q_{x_{j}}\left(\frac{1}{n_{0}} t\right)=\int_{-\infty}^{\infty} e^{\frac{i t x_{j}}{n_{0}}} \cdot f\left(x_{j}\right) d x_{j} \quad$ характеристическую функцию случайной величины $\frac{X_{j}}{n_{0}}\left(j=\overline{1, n_{0}}\right)$. Здесь $t-$ значение некоторой случайной величины $T$. Как будет показано далее, случайная величина $T_{1}=\frac{1}{\sqrt{2 \pi n_{0}}} T$ будет иметь нормальное распределение с $m_{t_{1}}=0$ и $\sigma_{t_{1}}=\sqrt{n_{0}}$.

Характеристическая функция случайной величины $\bar{x}$ запишется в виде 


$$
q_{x}(t)=\prod_{j=1}^{n_{0}} q_{x}\left(\frac{1}{n_{0}} t\right)=\prod_{j=1}^{n_{0}} \int_{-\infty}^{\infty} e^{\frac{i t x_{j}}{n_{0}}} \cdot f\left(x_{j}\right) d x_{j}
$$

так как величины $X_{j}$ независимы.

Не нарушая общности, можно положить, $m=0$.

Будем считать, что $\sigma=1$. Если это не так, то вместо $X_{j}\left(j=\overline{1, n_{0}}\right)$ будем рассматривать случайную величину $X_{j} / \sigma$. Очевидно если $\bar{x}$ будет иметь нормальное распределение, то $\frac{1}{\sigma} \bar{x}$ также будет иметь нормальное распределение.

Представим функцию $q_{x}\left(\frac{1}{n_{0}} t\right)$ рядом Маклорена.

Имеем $q_{x_{j}}(0)=1 ; q_{x_{j}}^{\prime}(0)=\frac{i}{n_{0}} \int_{-\infty}^{\infty} x_{j} f\left(x_{j}\right) d x_{j}=\frac{i}{n_{0}} m=0$.

Найдем вторую производную:

$$
q_{x}^{\prime \prime}(0)=\frac{i^{2}}{n_{0}^{2}} \int_{-\infty}^{\infty} x_{j}^{2} f(x) d x=-\frac{1}{n_{0}^{2}} \sigma^{2}=-\frac{1}{n_{0}^{2}} .
$$

Найдем остаточную сумму ряда $S_{\text {ост }}$ :

$$
S_{\text {ocr }}(t)=q_{x_{j}}(t)-1+\frac{1}{2 n_{0}^{2}} t^{2} .
$$

Поскольку $q_{x_{j}}(0)=1$, получаем $S_{\text {ост }}(0)=0$.

Следовательно,

$$
q_{x_{j}}(t)=1-\frac{1}{2 n_{0}^{2}} t^{2}
$$

Тогда

$$
q_{\bar{x}}(t)=\left(1-\frac{1}{2 n_{0}^{2}} t^{2}\right)^{n_{0}}
$$


Считаем, что $-3 \leq t \leq 3$ и $t^{2}=9<2 n_{0}^{2}$. Это допущение необходимо для определения функции $\frac{1}{\sqrt{2 \pi n_{0}}} q_{\bar{x}}(t)$. Следовательно,

$$
n_{0} \geq \sqrt{4,5} \text {. }
$$

Прологарифмируем выражение (2):

$$
\ln q_{\bar{x}}(t)=n_{0} \ln \left(1-\frac{1}{2 n_{0}^{2}} t^{2}\right) .
$$

Разложим полученное выражение в ряд Маклорена:

$$
\begin{aligned}
\ln q_{\bar{x}}(t) & =n_{0}\left(-\frac{1}{2 n_{0}^{2}} t^{2}-\frac{1}{4 n_{0}^{4} \cdot 2} t^{4}-\frac{1}{8 n_{0}^{8} \cdot 3} t^{8}-\ldots-\frac{1}{2^{k} n_{0}^{2 k} k} t^{2 k}-\ldots\right)= \\
& =-\frac{1}{2 n_{0}} t^{2}-\frac{1}{4 n_{0}^{3} \cdot 2} t^{4}-\frac{1}{8 n_{0}^{7} \cdot 3} t^{6}-\ldots-\frac{1}{2^{k} n_{0}^{2 k-1} k} t^{2 k} .
\end{aligned}
$$

Погрешность данного ряда не превосходит величины

$$
R_{x}(t)=\frac{\xi^{k+1} t^{k+1}}{2^{k+1} n_{0}^{2 k+1}(k+1)} \leq \frac{t^{2 k+2}}{2^{k+1} n_{0}^{2 k+1}(k+1)},
$$

так как $\xi \in(0, t)$. Потребуем, чтобы в формуле (4) при $k=2$ выполнялось условие

$$
\frac{t^{4}}{8 n_{0}^{3}} \leq \varepsilon_{0}
$$

где $\varepsilon_{0}$ - сколь угодно малое положительное число.

Поскольку $t \leq 3, \frac{3^{4}}{8 n_{0}^{3}} \leq \varepsilon_{0}$. Тогда

$$
n_{0} \geq\left\lfloor\frac{2,16}{3 \sqrt{\varepsilon_{0}}}\right\rfloor+1
$$


Из формул (3) и (5) находим

$$
n \geq n_{0}=\max \left\{\lfloor\sqrt{4,5}\rfloor+1,\left\lfloor\frac{2,16}{\sqrt[3]{\varepsilon}}\right\rfloor+1\right\} .
$$

Итак, с точностью $\varepsilon_{0} \ln q_{\bar{x}}(t)=-\frac{1}{2 n_{0}} t^{2}$, т.е.

$$
-\frac{1}{2 n_{0}} t^{2}-\varepsilon_{0} \leq \ln q_{\bar{x}}(t) \leq-\frac{1}{2 n_{0}} t^{2}+\varepsilon_{0} \text {. }
$$

Отсюда

$$
q_{x}(t)=e^{-\frac{1}{2 n_{0}} \sigma_{x}^{2} t^{2}}
$$

и

$$
e^{-\frac{1}{2 n_{0}} t^{2}} \cdot e^{-\varepsilon_{0}} \leq q_{\bar{x}}(t) \leq e^{-\frac{1}{2 n_{0}} t^{2}} \cdot e^{\varepsilon_{0}}
$$

Следовательно, относительная погрешность вычисления $q_{\bar{x}}(t)$ равна $e^{\varepsilon_{0}}-1=\varepsilon_{1}$.

При этом

$$
q_{\bar{x}}(t)=\int_{-\infty}^{\infty} e^{-i \bar{x}} \cdot f(\bar{x}) d \bar{x}
$$

Тогда $f(\bar{x})=\frac{1}{\sqrt{2 \pi}} \int_{-\infty}^{\infty} e^{-i \bar{x}} q_{\bar{x}}(t) d t$ и с учетом формулы (6) и формулы из работы [14] $\int_{-\infty}^{\infty} e^{A x^{2} \pm 2 B x-C} d x=\sqrt{\frac{\pi}{A}} e^{-\frac{A C-B^{2}}{A}}$ получаем

$$
f(\bar{x})=\frac{1}{\sqrt{2 \pi} / \sqrt{n_{0}}} \cdot e^{-\frac{\bar{x}^{2}}{2 / n_{0}}}
$$

Из формулы (6) следует, что $T_{1}$ имеет нормальное распределение и $\frac{1}{\sqrt{2 \pi n_{0}}} \int_{-\infty}^{\infty} q_{\bar{x}}(t) d t \approx \frac{1}{\sqrt{2 \pi n_{0}}} \int_{-3}^{3} q_{\bar{x}}(t) d t \approx 1$ с точностью 0,0027 . 
Имеем

$$
\begin{aligned}
f(\bar{x}) & =\frac{1}{2 \pi} \int_{-\infty}^{\infty} e^{-i \bar{x}} q_{\bar{x}}(t) d t \leq \frac{1}{2 \pi} \int_{-\infty}^{\infty} \cos t \bar{x} q_{\bar{x}}(t) d t-\frac{i}{2 \pi} \int_{-\infty}^{\infty} \sin t \bar{x} q_{\bar{x}}(t) d t \leq \\
& \leq \frac{1}{\pi} \int_{-\infty}^{\infty} q_{\bar{x}}(t) d t=\left(\frac{1}{\sqrt{2 \pi n_{0}}} \int_{-3}^{3} q_{\bar{x}}(t) d t\right) \frac{\sqrt{2 n_{0}}}{\sqrt{\pi}}=0,797 \sqrt{n_{0}} .
\end{aligned}
$$

Погрешность равна $0,797 \sqrt{n_{0}} \cdot 0,0027=0,0022 \sqrt{n_{0}}$.

Относительная погрешность $\varepsilon_{2}$ при этом составит $\frac{0,00227 \sqrt{n_{0}}}{0,797 \sqrt{n_{0}}}=$ $=0,0028$, т.e. $0,28 \%$.

Отметим, что $\varepsilon_{1}=e^{\varepsilon_{0}}-1>\varepsilon_{0}$.

Следовательно, можно утверждать, что с точностью $\varepsilon=\min \left\{\varepsilon_{0}, \varepsilon_{2}\right\}$ средняя арифметическая $n \geq n_{0}$ случайных величин при

$$
n_{0}=\max \left\{\lfloor\sqrt{4,5}\rfloor+1,\left\lfloor\frac{2,16}{\sqrt[3]{\varepsilon}}\right\rfloor+1\right\}
$$

имеет нормальное распределение.

В таблице для данного $\varepsilon$ показаны соответствующие значения $n_{0}$.

Зависимость $n_{0}$ от $\varepsilon$

\begin{tabular}{|c|c|c|c|c|c|c|c|c|c|c|c|c|}
\hline$\varepsilon$ & 0,001 & 0,0015 & 0,002 & 0,0028 & 0,003 & 0,004 & 0,005 & 0,01 & 0,1 & 0,5 & 0,7 & 0,9 \\
\hline$n_{0}$ & 23 & 20 & 18 & 16 & 16 & 15 & 14 & 11 & 6 & 4 & 3 & 3 \\
\hline
\end{tabular}

График зависимости $n_{0}$ от $\varepsilon$ представлен на рисунке.

Зависимость $n_{0}$ от $\varepsilon$ хорошо аппроксимируется полиномом шестой степени (получено с помощью средства MS Excel «Линия тренда») следующего вида:

$$
n_{0}=\left\lfloor-0,0007 \varepsilon^{6}+0,0291 \varepsilon^{5}-0,4377 \varepsilon^{4}+3,0656 \varepsilon^{3}-10,025 \varepsilon^{2}+18,508\right\rfloor .
$$

Коэффициент детерминации для полученной зависимости составляет $R^{2}=0,997$, что свидетельствует о хорошем подборе зависимости. 


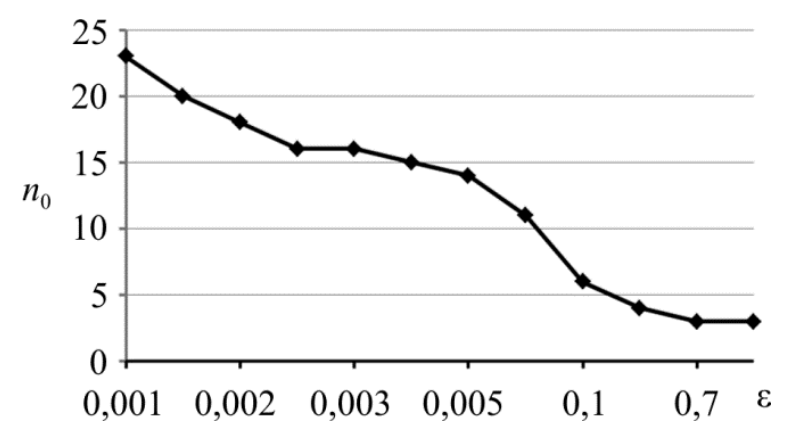

Рис. Зависимость $n_{0}$ OT $\varepsilon$

Следствие 1. Сумма $n$ независимых случайных величин, имеющих одинаковые математические ожидания и одинаковые дисперсии, имеет нормальный закон распределения при $n \geq n_{0}$, где $n_{0}$ удовлетворяет равенству (8).

Данное следствие, очевидно, следует из доказанной теоремы, поскольку если $\bar{x}$ имеет нормальное распределение при $n \geq n_{0}$, то $Y=n \bar{X}$ также будет иметь нормальное распределение при тех же значениях $n$.

Teорема 2. Средняя выборочная $n_{0}$ независимых случайных величин, математические ожидания которых попадают в один и тот же интервал $\left(m_{1}, m_{2}\right)$, а дисперсии попадают в один и тот же интервал $\left(\sigma_{1}^{2}, \sigma_{2}^{2}\right)$, имеет нормальное распределение при $n \geq \max \left\{n_{0}^{1}, n_{0}^{2}, n_{0}^{3}, n_{0}^{4}\right\}$, где $n_{0}^{1}, n_{0}^{2}, n_{0}^{3}, n_{0}^{4}$ удовлетворяют условию (8), при этом $n_{0}^{1}$ соответствует паре $\left(m_{1}, \sigma_{1}\right), n_{0}^{2}$ - паре $\left(m_{1}, \sigma_{2}\right), n_{0}^{3}$ - паре $\left(m_{2}, \sigma_{1}\right), n_{0}^{4}$ - паре $\left(m_{2}, \sigma_{2}\right)$.

Доказательство. Каждую случайную величину $X_{j}\left(j=\overline{1, n_{0}}\right)$ считаем нормированной случайной величиной. Далее используем метод, разработанный в теореме 1 для разных пар значений математического ожидания и среднего квадратического отклонения: $\left(m_{1}, \sigma_{1}\right),\left(m_{1}, \sigma_{2}\right)$, $\left(m_{2}, \sigma_{1}\right),\left(m_{2}, \sigma_{2}\right)$. Находим соответствующие значения $n_{0}^{1}, n_{0}^{2}, n_{0}^{3}, n_{0}^{4}$. Из них выбираем максимальное, которое будет определять необходимое число слагаемых, определяющих нормально распределенную среднюю выборочную. 
Следствие 2. Сумма $n_{0}$ независимых случайных величин, математические ожидания которых попадают в один и тот же интервал $\left(m_{1}, m_{2}\right)$, а дисперсии попадают в один и тот же интервал $\left(\sigma_{1}^{2}, \sigma_{2}^{2}\right)$, имеет нормальное распределение при $n \geq \max \left\{n_{0}^{1}, n_{0}^{2}, n_{0}^{3}, n_{0}^{4}\right\}$, где $n_{0}^{1}, n_{0}^{2}, n_{0}^{3}, n_{0}^{4}$ удовлетворяют условию теоремы 2 .

\section{Заключение}

В статье доказаны две теоремы и получены два следствия из них. Получены формульные условия для необходимого числа слагаемых, при которых средняя выборочная и суммы слагаемых центральной предельной теоремы будут иметь с заданной точностью нормальный закон распределения.

Дальнейшим развитием исследований является определение необходимого числа слагаемых для зависимых случайных величин, а также случайных векторов и матриц.

\section{Список литературы}

1. Золотарев В.М. Современная теория суммирования независимых случайных величин. - М.: Наука, 1986. - 415 с.

2. Сенаторов В.В. О реальной точности аппроксимаций в центральной предельной теореме // Сибирский математический журнал. - 2011. - Т. 52, № 4. - С. 913-935.

3. Шевцова И.Г. Точность нормальной аппроксимации: методы оценивания и новые результаты. - М.: АРГАМАК-МЕДИА, 2016. - 380 с.

4. Нефедова Ю.С., Шевцова И.Г. О неравномерных оценках скорости сходимости в центральной предельной теореме // Теория вероятностей и ее применения. - 2012. - Т. 57, № 1. - С. 62-97.

5. Королев В.Ю., Попов С.В. Уточнение оценок скорости сходимости в центральной предельной теореме при отсутствии моментов порядков, больших второго // Теория вероятностей и ее применения. - 2011. - Т. 56, № 4. - С. 797-805.

6. Попов С.В. Уточнение неравномерных оценок скорости сходимости в центральной предельной теореме при существовании моментов не выше второго // Информатика и ее применения. - 2012. - Т. 6, № 1. - С. 7-11.

7. Гринь А.Г. О центральной предельной теореме для симметрических функций от зависимых величин // Математические структуры и моделирование. - 2017. - № 1(41). - С. 5-11. 
8. Волгин А.В. Оценка скорости сходимости в многомерной центральной предельной теореме // Прикладная дискретная математика. - 2017. № 36. - C. 13-24.

9. Гетце Ф., Наумов А.А., Тихомиров А.Н. Предельные теоремы для двух классов случайных матриц с зависимыми элементами // Теория вероятностей и ее применения. - 2014. - Т. 59, № 1. - С. 61-80.

10. Резников В.М. К методологии проверки адекватности центральной предельной теоремы // Философия науки. - 2012. - № 3 (54). - С. 81-91.

11. Пименов С.Ю., Тинаев В.В. Применение центральной предельной теоремы для компьютерного моделирования случайных сигналов // Наука и образование: новое время. - 2017. - № 2 (19). - С. 227-231.

12. Парахин А.С. Численная проверка центральной предельной теоремы // Математика, информатика, компетентностный подход к обучению в вузе и школе: материалы Всерос. науч.-практ. конф., г. Курган, 14 апреля 2015 г. - Курган: Изд-во КГУ, 2015. - С. 24-27.

13. Цурганов А.Г., Макеенко Г.И. Простая иллюстрация центральной предельной теоремы в медицинской статистике // Достижения фундаментальной, клинической медицины и фармации: материалы 71-й науч. сессии сотр. ун-та, г. Витебск, 27-28 января 2016 г. / ВГМУ. - Витебск, 2016. C. $330-331$.

14. Вентцель Е.С. Теория вероятностей: учеб. - М.: Изд-во физ.-мат. лит., 2003. -564 с.

15. Ганичева А.В. Теория вероятностей: учеб. пособие. - СПб.: Лань, 2017. - $144 \mathrm{c}$.

\section{References}

1. Zolotarev V.M. Sovremennaia teoriia summirovaniia nezavisimykh sluchainykh velichin [Modern theory of summation of independent random variables]. Moscow, Nauka, 1986,415 p.

2. Senatorov V.V. O real'noi tochnosti approksimatsii $\mathrm{v}$ tsentral'noi predel'noi teoreme [On the real accuracy of approximations in the Central limit theorem]. Siberian Mathematical Journal, 2011, V. 52, no. 4, pp. 913-935.

3. Shevtsova I.G. Tochnost' normal'noi approksimatsii: metody otsenivaniia i novye rezul'taty [Accuracy of normal approximation: estimation methods and new results]. Moscow, ARGAMAK-MEDIA, 2016, 380 p.

4. Nefedova Iu.S., Shevtsova I.G. O neravnomernykh otsenkakh skorosti skhodimosti $\mathrm{v}$ tsentral'noi predel'noi teoreme [On uneven estimates of the convergence rate in the Central limit theorem]. Theory of Probability and its Applications, 2012, V. 57, no. 1, pp. 62-97. 
5. Korolev V.Iu., Popov C.B. Utochnenie otsenok skorosti skhodimosti v tsentral'noi predel'noi teoreme pri otsutstvii momentov poriadkov, bol'shikh vtorogo [Refinement of convergence rate estimates in the Central limit theorem in the absence of moments of orders greater than the second]. Theory of Probability and its Applications, 2011, V. 56, no. 4, pp. 797-805.

6. Popov S.V. Utochnenie neravnomernykh otsenok skorosti skhodimosti $\mathrm{v}$ tsentral'noi predel'noi teoreme pri sushchestvovanii momentov ne vyshe vtorogo [Refinement of uneven estimates of the convergence rate in the Central limit theorem for the existence of moments no higher than the second]. Informatics and Applications, 2012, V. 6, no. 1, pp. 7-11.

7. Grin' A.G. O tsentral'noi predel'noi teoreme dlia simmetricheskikh funktsii ot zavisimykh velichin [On the Central limit theorem for symmetric functions on dependent variables]. Mathematical Structures and Modeling, 2017, no. 1(41), pp. 5-11.

8. Volgin A.V. Otsenka skorosti skhodimosti v mnogomernoi tsentral'noi predel'noi teoreme [Estimation of the convergence rate in the multidimensional Central limit theorem]. Prikladnaia diskretnaia matematika, 2017, no. 36, pp. 13-24.

9. Gettse F., Naumov A.A., Tikhomirov A. N. Predel'nye teoremy dlia dvukh klassov sluchainykh matrits s zavisimymi elementami [Limit theorems for two classes of random matrices with dependent elements]. Theory of Probability and its Applications, 2014, V. 59, no. 1, pp. 61-80.

10. Reznikov V.M. K metodologii proverki adekvatnosti tsentral'noi predel'noi teoremy [On the methodology for checking the adequacy of the Central limit theorem]. Filosofiia nauki, 2012, no, 3 (54), pp. 81-91.

11. Pimenov S.Iu. Tinaev V.V. Primenenie tsentral'noi predel'noi teoremy dlia komp'iuternogo modelirovaniia sluchainykh signalov [Application of the Central limit theorem for computer simulation of random signals]. Nauka i obrazovanie: novoe vremia, 2017, 2 (19), pp. 227-231.

12. Parakhin A.S. Chislennaia proverka tsentral'noi predel'noi teoremy [Numerical verification of the Central limit theorem]. Matematika, informatika, kompetentnostnyi podkhod $k$ obucheniiu v vuze i shkole. Materialy Vseros. nauch.prakt. konf., g. Kurgan, 14 apr. 2015. Kurgan, izd.-vo KGU, 2015, pp. 24-27.

13. Tsurganov A.G., Makeenko G.I. Prostaia illiustratsiia tsentral'noi predel'noi teoremy v meditsinskoi statistike [A simple illustration of the Central limit theorem in medical statistics]. Dostizheniia fundamental'noi, klinicheskoi meditsiny i farmatsii: materialy 71-i nauch. ses. sotr. un-ta, g. Vitebsk 27-28 ianv. 2016, Vitebsk: VGMU, 2016, pp. 330-331.

14. Venttsel' E.S. Teoriia veroiatnostei: Uchebnik [Probability theory: Textbook]. Moscow, Izdatel'stvo fiziko-matematicheskoi literatury, 2003,. 564 p. 
15. Ganicheva, A.V. Teoriia veroiatnostei: Uchebnoe posobie [Probability theory: a tutorial]. Saint Petersburg, «Lan'», 2017, 144 p.

Статья получена: 04.09.2020

Статья принята: 16.11.2020

\section{Сведения об авторе}

Ганичева Антонина Валериановна (Тверь, Россия) - кандидат физико-математических наук, доцент, доцент кафедры «Физико-математические дисциплины и информационные технологии», Тверская государственная сельскохозяйственная академия (170904, Тверь, ул. Маршала Василевского, 7, e-mail: alexej.ganichev@yandex.ru).

\section{About the author}

Antonina V. Ganicheva (Tver, Russian Federation) - Ph.D. in Physics and Mathematics, Associate Professor, Department of Physical and Mathematical Disciplines and Information Technologies, Tver State Agricultural Academy (7, Marshal Vasilevsky st., Tver, 170904, e-mail: alexej.ganichev@yandex.ru).

\section{Библиографическое описание статьи согласно ГОСТ Р 7.0.100-2018:}

Ганичева, А.В. Оценка числа слагаемых центральной предельной теоремы / A.B. Ганичева. - DOI: 10.15593/2499-9873/2020.4.01. - Текст: непосредственный // Прикладная математика и вопросы управления = Applied Mathematics and Control Sciences. -2020 . - № 4. - C. 7-19.

\section{Цитирование статьи в изданиях РИНЦ:}

Ганичева А.В. Оценка числа слагаемых центральной предельной теоремы // Прикладная математика и вопросы управления. - 2020. - № 4. - С. 7-19. DOI: $10.15593 / 2499-9873 / 2020.4 .01$

\section{Цитирование статьи в references и международных изданиях:}

\section{Cite this article as:}

Ganicheva A.V. Estimation of the number of summands of the central limit theorem. Applied Mathematics and Control Sciences, 2020, no. 4, pp. 7-19. DOI: 10.15593/24999873/2020.4.01 (in Russian) 